\title{
Desempenho reprodutivo de ovelhas mestiças lanadas e deslanadas submetidas a protocolo hormonal a base de progestágeno e eCG, durante a contraestação reprodutiva
}

\section{Reproductive performance of woolly and hairless crossbred ewes treated with an exogenous progestagen and $\mathrm{eCG}$ hormone during the non-breeding season}

\author{
Gustavo Martins Gomes dos Santos ${ }^{1 *}$; Katia Cristina Silva-Santos ${ }^{1}$; Fabiana \\ Andrade Melo-Sterza ${ }^{2}$; Ivone Yurika Mizubuti ${ }^{3}$; Fernanda Barros Moreira ${ }^{4}$; \\ Marcelo Marcondes Seneda ${ }^{5}$
}

\section{Resumo}

Este trabalho teve como objetivo avaliar o desempenho reprodutivo de ovelhas mestiças lanadas e deslanadas submetidas a um protocolo hormonal a base de progestágeno e eCG durante a contraestação reprodutiva. Ovelhas mestiças $(\mathrm{n}=48)$, sem raça definida, foram divididas em dois tratamentos, considerando-se a presença (G-Lanada, $n=25)$ ou ausência de lã (G-Deslanada, n=23). As ovelhas foram submetidas a um tratamento hormonal de indução/sincronização de estro, que consistiu na colocação do dispositivo intravaginal em dia aleatório do ciclo estral (D0). No D7, foi administrado eCG e d-cloprostenol. No D9, o dispositivo foi retirado e após $12 \mathrm{~h}$ os machos foram introduzidos no lote (proporção 1:6), nos dias 10, 11 e 12. Posteriormente, os machos foram separados das fêmeas por dez dias e então reintroduzidos no lote por mais 45 dias. A taxa de apresentação de estro e de prenhez resultantes da sincronização foi 84,0 e $36,0 \%$ vs. 87,0 e $56,6 \%$, G-Lanada vs. G-Deslanada (p>0,05). A taxa de prenhez total, após repasse com os reprodutores foi 68,0 vs. 91,3\%, G-Lanada vs. G-Deslanada $(\mathrm{p}<0,05)$. Conclui-se que ovelhas mestiças deslanadas tratadas com protocolo hormonal durante a contraestação apresentaram desempenho reprodutivo superior ao de ovelhas mestiças lanadas.

Palavras-chave: Indução/sincronização de estro. Lanadas. Deslanadas. Ovelhas. Contraestação reprodutiva.

\begin{abstract}
The aim of this study was to evaluate the reproductive performance of woolly and hairless crossbred ewes treated with an exogenous progestagen and eCG hormone during the non-breeding season. Mixed breed ewes $(n=48)$ were assigned into two treatments considering the presence (G-Woolly, $n$ $=25$ ) or absence of wool (G-Hairless, $\mathrm{n}=23$ ). The ewes underwent hormone treatment to induction/
\end{abstract}

1 Doutorando do Programa de Pós-Graduação em Ciência Animal. Universidade Estadual de Londrina, UEL. E-mail: gugamgs@ hotmail.com; kati_cris@hotmail.com

2 Pror $^{\mathrm{a}} \mathrm{Dr}^{\mathrm{a}}$ da Universidade Estadual do Mato Grosso do Sul, UEMS, Unidade de Aquidauana. E-mail: fabi_melo@hotmail.com

3 Prof ${ }^{a} \operatorname{Dr}^{\mathrm{a}}$ do Departamento de Zootecnia, Centro de Ciências Agrárias, Universidade Estadual de Londrina, UEL. Bolsista de Porudtividade do CNPq. E-mail: mizubuti@uel.br

4 Dr $^{\mathrm{a}}$ Pesquisadora. Universidade Estadual de Londrina, UEL. E-mail: fbmoreira@sercomtel.com.br

5 Prof. Dr. do Departamento de Clínicas Veterinárias, Centro de Ciências Agrárias, Universidade Estadual de Londrina DCV/CCA/ UEL. Bolsista de Porudtividade do CNPq. E-mail: mseneda@uel.br

* Autor para correspondência

Recebido para publicação $11 / 06 / 2010$ Aprovado em 03/05/201 1 
synchronization of estrus, which consisted of the insertion of an intravaginal device randomly at the estrous cycle (D0). On Day 7, ewes were injected with eCG and d-cloprostenol. On Day 9, the device was removed and males were introduced into the herd (proportion of 1:6) twelve hours later during days 10, 11 and 12. After Day 12, males were separated from females for ten days and later reintroduced into the herd for 45 days. The rate of onset of estrus and the pregnancy rate from the synchronization was 84.0 and $36.0 \%$ vs. 87.0 and $56.6 \%$, G-Woolly vs. G-Hairless ( $>0.05$ ). The total pregnancy rate after male reintroduction was 68.0 vs. $91.3 \%$, G-Woolly vs. G-Hairless $(\mathrm{p}<0.05)$. In conclusion hairless crossbred ewes treated with the exogenous progestagen and $\mathrm{eCG}$ hormone during the non-breeding season presented higher reproductive performance compared to woolly crossbred ones.

Key words: Ewes. Woolly. Hairless. Estrus induction/synchronization. Non-breeding season.

\section{Introdução}

A maioria das raças de ovinos apresenta um modelo de reprodução sazonal com incidência de ciclos estrais concentrados durante o outono e inverno, período de luminosidade decrescente. Já nos períodos de luminosidade crescente, conhecido como período de contraestação reprodutiva, os ovinos apresentam graus variados de estacionalidade reprodutiva (SASA et al., 2001; HAFEZ; JAINUDEEN; WAHID, 2004). Um dos principais fatores responsáveis por esta estacionalidade é o fotoperíodo, cuja influência na reprodução das fềmeas e dos machos é interdependente da latitude, da raça e das condições nutricionais (ROSA; BRYANT, 2003; SASA et al., 2002; MORI et al., 2006).

O Brasil, país de dimensões continentais, apresenta variação considerável na duração da estação reprodutiva das ovelhas (SASA et al., 2002). É possível observar grande diferença entre as raças lanadas, normalmente criadas no Sul, e as deslanadas, geralmente criadas na região Nordeste do país. A produção de cordeiros anual é maior nos rebanhos de raças deslanadas, pois além de serem poliéstricas anuais, apresentam maior taxa de ovulação e, consequentemente, maiores índices de prolificidade do que as ovelhas lanadas (VILLARROEL, 1991). Intervalo médio entre partos de oito meses foi relatado para raças deslanadas, contrastando com o período médio de doze meses para raças lanadas (COSTA et al., 1990).

$\mathrm{Na}$ Região Nordeste, as ovelhas deslanadas ciclam ao longo do ano, podendo ser acasaladas mais de uma vez ao ano (FIGUEIREDO; OLIVEIRA; BELLAVER, 1980; GIRÃO; MEDEIROS; GIRÃO, 1984). Nesta região, a estacionalidade da atividade reprodutiva varia mais em função da temperatura e da nutrição do que efetivamente devido ao fotoperíodo (SIMPLÍCIO et al., 1982; SILVA; NUNES, 1987; SILVA et al., 1987). Nas Regiões Sul e Sudeste, trabalhos realizados com raças de duplo propósito (lã e carne) e especializadas para produção de carne apresentaram uma estação reprodutiva mais restrita à estação de outono (PRUCOLLI; BACCARI JUNIOR, 1967; NUNES; FIGUEIRÓ, 1975; SILVA; FIGUEIRÓ, 1980; RODA; et al., 1993; RIBEIRO; ROCHA; SILVA, 1996).

Embora exista diferença entre as ovelhas lanadas e deslanadas, em relação às características reprodutivas, poucos são os trabalhos que avaliam estas diferenças em relação a animais mestiços, cruzados, sem raça definida, sendo este tipo de animal bem característico da maioria das propriedades brasileiras.

Devido a esta estacionalidade reprodutiva apresentada por algumas raças ovinas, nos últimos anos, alguns grupos de pesquisa têm buscado alternativas para promover a indução/sincronização de estro em ovelhas ao longo do ano. Efetiva indução/sincronização do estro tem sido obtida em pequenos ruminantes pelo uso de dispositivos de progesterona/progestágenos, associados ou não a eCG, FSH, PGF2 a e GnRH. Exemplos disso são as esponjas intravaginais impregnadas com progestágenos, tais como acetato de fluorogestona (FGA) e acetato de medroxiprogesterona (MAP) (KUSAKARI; OHARA; MORI, 1995; MUFTI; 
WANI; WANI, 1997; GODFREY et al., 1999; UNGERFELD; RUBIANES, 2002; KOHNO et al., 2005; DOGAN; NUR, 2006). Como vantagens desta técnica, podemos citar a concentração dos cios, concentração da mão-de-obra, indução da ciclicidade de fêmeas em anestro, diminuição do intervalo entre partos, melhor utilização do reprodutor, altas taxas de prenhez no início das estações de monta, homogeneização dos lotes com maiores ganhos na comercialização; tudo isto levando a um aumento da eficiência reprodutiva (HENDERSON; DOWNING; BECK, 1984).

Devido à falta de informações sobre as características reprodutivas de ovelhas mestiças lanadas e deslanadas, o objetivo deste trabalho foi avaliar a taxa de indução/sincronização de estro, taxa de prenhez e de prolificidade de ovelhas submetidas a um protocolo hormonal a base de progestágeno e eCG durante a contraestação reprodutiva.

\section{Material e Métodos}

O experimento foi conduzido em uma propriedade localizada no norte do estado do Paraná, Brasil (23 $\square 18$ " de latitude sul, 51 $\square 09$ " de longitude W-GR) nos meses de setembro a dezembro. O clima predominante no local é o subtropical, com concentração de chuvas no verão. As temperaturas médias variam de $27 \square \mathrm{C}$, no verão, a $15 \square \mathrm{C}$, no inverno. O período de luminosidade local média durante o período experimental foi 11:57, 12:38, 13:15, 13:34 horas para os meses de setembro, outubro, novembro e dezembro, respectivamente (ANUÁRIO INTERATIVO DO OBSERVATÓRIO NACIONAL, 2009).

Durante o período experimental, os animais foram mantidos em pastagem de 11,4 hectares, composta de grama Estrela Roxa (Cynodon pletostachyrus Pilger), Brachiaria decumbens e grama Mato Grosso (Paspalum notatum), sob pastejo contínuo, com acesso a sal mineral e água ad libitum. À noite, os animais eram levados para o aprisco e, pela manhã, era fornecido $400 \mathrm{~g} /$ dia de casquinha de soja por animal.

\section{Animais e Tratamentos}

O delineamento experimental foi inteiramente casualizado com quarenta e oito ovelhas mestiças, sem raça definida, não gestantes, com idade média de $2 \pm 1$ ano, com escore de condição corporal médio de 3,2 $\pm 0,5$, escala de 1 a 5 (CALDEIRA; VAZ PORTUGAL, 1998), e peso vivo médio de 41 $\pm 2 \mathrm{Kg}$.

Os animais foram divididos em dois grupos experimentais, considerando-se a presença ou ausência de lã, sendo, G-Lanada (n=25), ovelhas com o corpo totalmente coberto por lã, e G-Deslanada $(n=23)$, ovelhas com o corpo completamente desprovido de lã. Ambos os grupos foram submetidos a um protocolo hormonal de indução/sincronização de estro, que consistiu na colocação de um dispositivo intravaginal contendo $60 \mathrm{mg}$ de Acetato de Medroxiprogesterona (MAP; Progespon $^{\circledR}$, Syntex, Argentina), em dia aleatório do ciclo estral denominado de D0. No D7, foi administrado 300 UI de eCG (Novormon ${ }^{\circledR}$, Syntex, Argentina) e $30 \mu \mathrm{g}$ de d-Cloprostenol (Prolise ${ }^{\circledR}$, Arsa S.R.L., Argentina), via intramuscular. No D9, o dispositivo foi retirado e, após 12 horas, os machos foram introduzidos no lote em uma proporção de 1:6. Os acasalamentos foram realizados das 07 às 09 horas e das 16 às 18 horas, durante os dias experimentais (D10, 11 e 12). Após o D12, os machos foram isolados das fêmeas por dez dias e depois reintroduzidos para repasse por um período de 45 dias (Figura 1).

Para a realização das observações de estro e montas naturais, foram utilizados carneiros Santa Inês $(n=6)$ e Suffolk $(n=2)$, selecionados após exame andrológico (CBRA, 1998).

Para a avaliação da taxa de apresentação de estro, consideraram-se as ovelhas que apresentaram sinais de estro e permitiram serem acasaladas pelos machos nos dias experimentais D10, 11 e 12 . O diagnóstico 
de gestação foi realizado por ultrassonografia transretal (Aloka SSD 500, transdutor linear de 5 $\mathrm{MHz}$ ), 30 dias após o último dia de acasalamento dos estros induzidos/sincronizados e, igualmente,
30 dias após a retirada definitiva dos machos, a fim de diferenciar as prenhezes oriundas dos acasalamentos dos dias experimentais D10, 11 e 12 e do período de repasse.

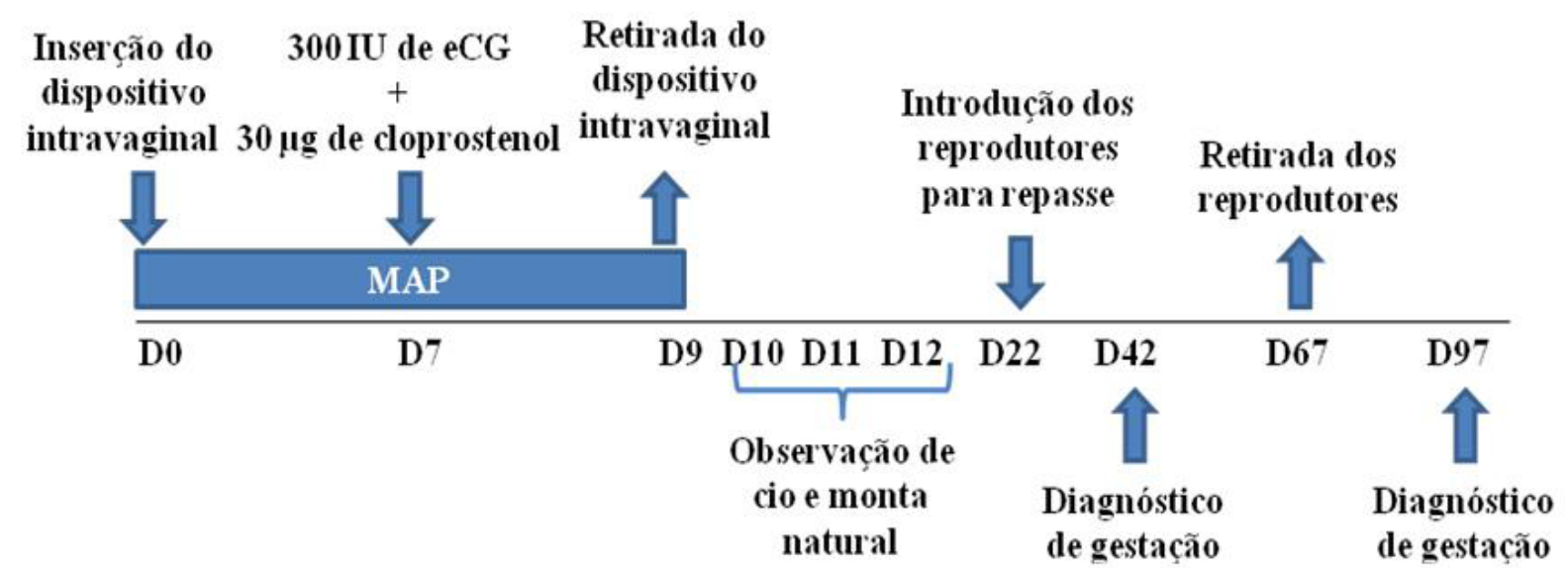

Figura 1. Diagrama esquemático do protocolo hormonal a base de progestágeno utilizado em ovelhas mestiças lanadas e deslanadas durante a contraestação reprodutiva.

\section{Variáveis Analisadas}

Os parâmetros avaliados foram: taxa de apresentação de estro resultante da observação nos dias experimentais D10, 11 e 12; intervalo entre a retirada do progestágeno e o início do estro; duração do estro; taxa de prenhez à sincronização (resultante dos acasalamentos dos dias experimentais D10, $11 \mathrm{e}$ 12); taxa de prenhez total, ao final do período de 45 dias de estação de monta e a prolificidade (número de cordeiros nascidos por ovelha parida em cada tratamento).

\section{Análise Estatística}

A duração média do estro foi analisada pelo teste de ANOVA e o desempenho reprodutivo foi analisado pelo teste de Qui-Quadrado, utilizando o programa Bioestat 5.0 (AYRES et al., 2007), considerando um nível de significância de 5\%.

\section{Resultados e Discussão}

A sazonalidade reprodutiva é uma característica importante, que limita a produtividade dos pequenos ruminantes (ZARAZAGA; MALPAUX;
CHEMINEAU, 2003). Esta estacionalidade é influenciada por muitos fatores, que podem estar associados ou não, entre eles o fotoperíodo (ROSA; BRYANT, 2003), a latitude (SASA et al., 2002), a nutrição (MORI et al., 2006) e a raça dos animais (FONSECA, 2005).

Neste trabalho, observou-se que ovelhas mestiças deslanadas submetidas a um protocolo hormonal a base de progestágeno apresentaram desempenho reprodutivo superior às lanadas, ao final do período de estação de monta realizado durante a contraestação reprodutiva.

Das 48 ovelhas utilizadas no experimento, $85,42 \%$ (41/48) apresentaram estro durante o período de observação nos dias experimentais D10, 11 e 12. Independente da característica racial, não houve diferença em relação à taxa de apresentação de estro entre os dois grupos experimentais (84\% - G-Lanada; 87\% - G-Deslanada, p>0,05), após protocolo hormonal. Resultados semelhantes, foram relatados para ovelhas deslanadas (DIAS et al., 2001; RODRIGUES et al., 2008; SILVA, 2008) e lanadas (KUSAKARI; OHARA; MORI, 1995; TAKADA et al., 2003; SALAZAR, 2006; GRECCO; MONREAL, 2008), cuja taxa de 
apresentação de estro variou de 73,5 a 100\%, durante o período de estacionalidade reprodutiva. Estes resultados demonstram que os tratamentos hormonais de indução/sincronização de estro a base de progestágenos, eCG e PGF2 $\alpha$ são eficientes em induzir estro tanto em ovelhas lanadas como em deslanadas, durante o período da contraestação reprodutiva.

Não houve diferença entre os grupos experimentais em relação ao tempo entre a retirada do progestágeno e o início do estro ( $p>0,05$; Figura 2). Foi observado que ovelhas mestiças lanadas apresentaram maior concentração de estro entre 36 e 48 horas após a retirada do dispositivo de progesterona, concordando com os resultados observados por Takada et al. (2003). Porém, Salazar (2006) observou maior concentração dos estros 60 horas após a retirada do dispositivo. As ovelhas mestiças deslanadas apresentaram maior concentração dos estros 48 horas após a retirada do progestágeno, o que concorda com os resultados observados por Dias et al. (2001). Ovelhas lanadas apresentaram tempo menor de duração do estro (21 horas), quando comparado às ovelhas deslanadas (30 horas, $\mathrm{p}<0,05$ ). Outros autores, trabalhando em condições semelhantes, observaram durações médias de estro de 21,8 (FUKUI et al.,1991) e 23,1 (IIDA et al., 2004) horas para ovelhas lanadas.

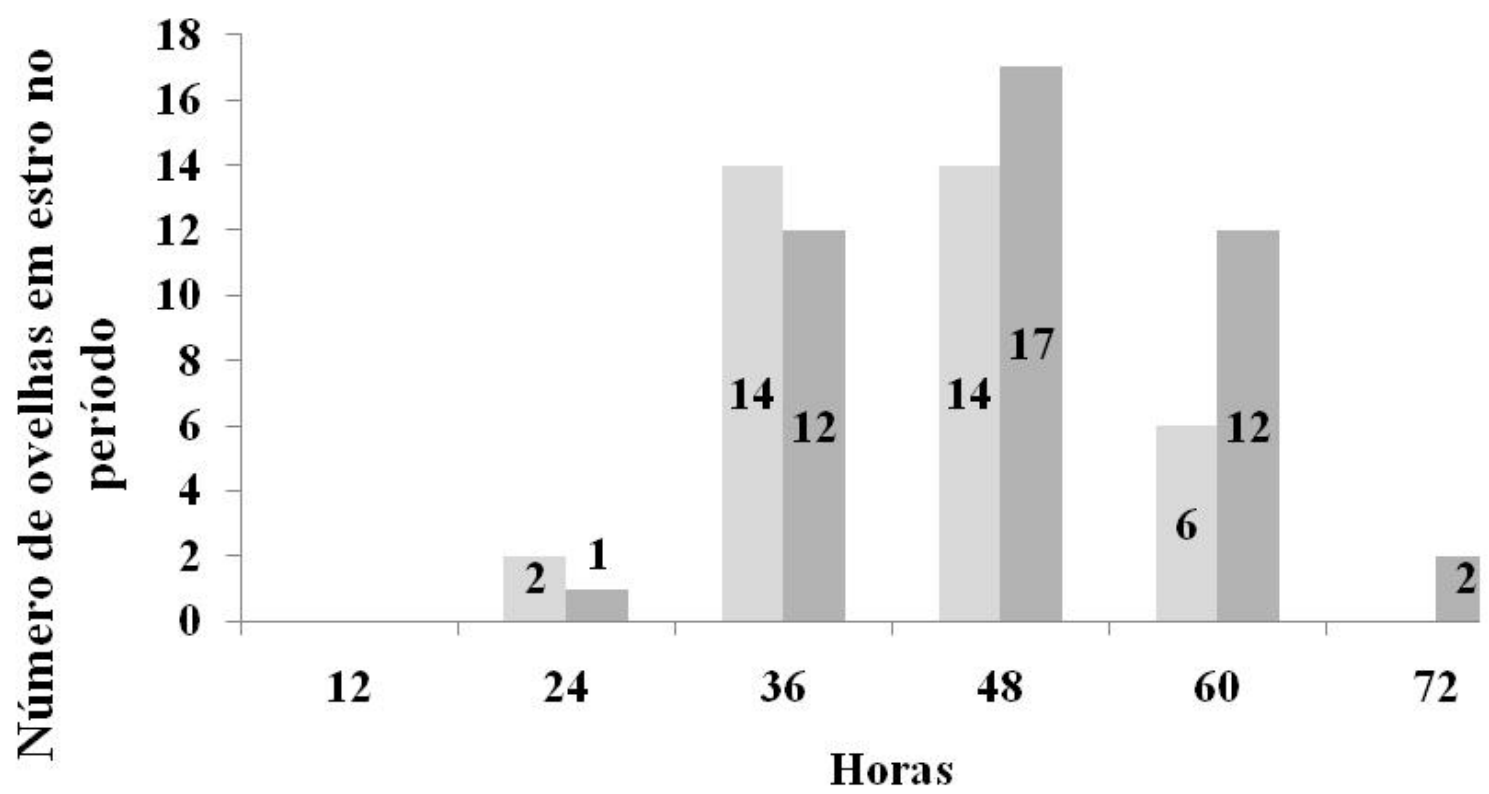

\section{G-I anada G-Deslanada}

Figura 2. Número de ovelhas mestiças lanadas e deslanadas, submetidas a um protocolo hormonal a base de progestágeno durante o período de contraestação reprodutiva, que foram detectadas em estro por período de observação, durante os dias experimentais D10, 11 e $12, \mathrm{p}>0,05$.

Estudo avaliando a ciclicidade de ovelhas lanadas e deslanadas demonstrou diferença na taxa de apresentação de estro e na concentração de progesterona entre os dois grupos raciais, ao longo do ano. As borregas deslanadas da raça Santa Inês apresentaram atividade cíclica reprodutiva entre os meses de abril a novembro e as lanadas das raças
Romney Marsh e Suffolk, num período mais curto entre os meses de abril e julho, apresentando-se em anestro no período de agosto a novembro, período de estacionalidade reprodutiva ou contraestação reprodutiva no Brasil (SASA et al., 2002).

A taxa de prenhez à sincronização, resultante do protocolo hormonal de indução/sincronização 
de estro durante o período da contraestação apresentada neste trabalho para as ovelhas lanadas (36\%, Tabela 1) é semelhante à encontrada por outros pesquisadores: 41,2\% (DOGAN; NUR, 2006) e 55\% (KOHNO et al., 2005), porém, menor em relação à taxa de prenhez de ovelhas Suffolk $(73,53 \%)$, submetidas a tratamento hormonal durante a estacionalidade reprodutiva (SALAZAR, 2006). Para ovelhas deslanadas, a taxa de prenhez de $56,5 \%$ apresentada no presente trabalho (Tabela 1) é semelhante à de ovelhas Santa Inês $(63,8 \%)$, após protocolo de indução/sincronização de estro, durante a contraestação reprodutiva (FIGUEIREDO et al., 2007). A diferença entre as taxas de apresentação de estro e de prenhez observada nos diversos trabalhos deve-se, provavelmente, ao tipo de protocolo utilizado, à raça e às condições nutricionais das fêmeas (OZYURTLU; KUCUKASLAN; CETIN, 2010).

Tabela 1. Taxa de prenhez à sincronização, taxa de prenhez total e ao final do período de 45 dias de estação de monta e prolificidade de ovelhas mestiças lanadas e deslanadas, submetidas a um protocolo hormonal a base de progestágeno durante o período de contraestação reprodutiva.

\begin{tabular}{lccc}
\hline & G-Lanada & G-Deslanada & Valor de P \\
\hline Número de Ovelhas & 25 & 23 & - \\
Taxa de Prenhez à Sincronização $(\%)$ & $36,0(21 / 25)$ & $56,5(13 / 23)$ & 0,16 \\
Taxa de Prenhez Total (\%) & $68,0(17 / 25)^{\mathrm{a}}$ & $91,3(21 / 23)^{\mathrm{b}}$ & 0,047 \\
Prolificidade & $1,18(20 / 25)$ & $1,05(22 / 23)$ & 0,20 \\
\hline
\end{tabular}

${ }^{\mathrm{a}} \mathrm{e}^{\mathrm{b}}$ na mesma linha diferem a $5 \%$ de probabilidade.

No presente trabalho, foi observado que, da mesma maneira que existem diferenças na atividade reprodutiva de ovelhas lanadas e deslanadas de raças puras (SASA et al., 2002; ROSA; BRYANT, 2003; IIDA et al., 2004; DOGAN; NUR, 2006; MORI et al., 2006), durante o período de contraestação reprodutiva, o mesmo ocorre com ovelhas mestiças, sem raça definida. Não foi observado diferença em relação à taxa de prenhez após o protocolo hormonal, porém, em relação à taxa de prenhez total, ao final da estação de monta, a diferença foi de vinte e três pontos percentuais a favor das ovelhas mestiças deslanadas $(91,3 \%)$ em relação às lanadas $(68,0 \%, \mathrm{p}<0,05$; Tabela 1). Também observou-se que, mesmo havendo diferença, a taxa de prenhez de ovelhas mestiças lanadas ao final do período de estação de monta sugere que animais mestiços apresentam certo grau de atividade reprodutiva durante o período de contraestação e não um completo estado de anestro.

Duas hipóteses podem ser aventadas para explicar esta taxa de prenhez apresentada pelas ovelhas mestiças lanadas ao final do período de estação de monta. Primeiramente, o protocolo hormonal pode ter proporcionado, em algumas fêmeas, a indução da ciclicidade, possibilitando que estas retornassem em estro durante o período de repasse. Outra hipótese seria que para estes animais sem raça definida a estacionalidade reprodutiva seja mais influenciada pelas condições de manejo e nutrição do que efetivamente pelo fotoperíodo.

Santos et al. (2009), trabalhando com ovelhas mestiças lanadas e deslanadas, observaram que os animais submetidos a suplementação alimentar, durante o período da contraestação, apresentaram uma maior taxa de prenhez $(81,8 \%)$, quando comparada as que não foram suplementadas $(41,7 \%)$, demonstrando que o fator nutricional influencia a estacionalidade reprodutiva das ovelhas mestiças.

Ao analisar o desempenho reprodutivo de ovelhas, Gatenby (1986) cita que algumas raças 
deslanadas, indígenas, dos trópicos exibem estro em todos os meses do ano, embora uma proporção delas, sob condições desfavoráveis, apresente variações estacionais de estro devido, primariamente, a mudanças sazonais no fornecimento de alimentos. Da mesma maneira, rebanhos africanos lanados, experimentais e bem alimentados apresentam estro ao longo de todo ano, enquanto rebanhos nômades e mal alimentados não apresentam cio na estação seca e quente devido à má nutrição.

Não foi observada diferença na prolificidade das ovelhas entre os dois grupos experimentais: 1,18 - G-Lanada e 1,05 - G-Deslanada (Tabela 1). Os resultados obtidos foram semelhantes ao de ovelhas Suffolk, submetidas à indução/sincronização de estro, durante o período de estacionalidade reprodutiva: 1,08 (SALAZAR, 2006) e 1,03 (GRECCO; MONREAL, 2008). Elias, Cohen e Dayenoff (1985) notaram que acasalamentos de ovinos deslanados da raça Dorper realizados durante a primavera e o verão resultaram no nascimento de 1,52 a 1,57 cordeiro/parto. A prolificidade reportada para ovinos deslanados (1,73 - Dorper) e lanados (1,40 - Merino), sob mesmas condições de manejo (CLOETE; SNYMAN; HERSELMAN, 2000), foram superiores aos encontrados no presente estudo para ambos grupos (1,18 e 1,05 cordeiro/parto).

\section{Conclusão}

Conclui-se que ovelhas mestiças deslanadas sem raça definida, submetidas a protocolo hormonal a base de progestágeno, d-cloprostenol e eCG durante a contraestação reprodutiva apresentaram desempenho reprodutivo superior ao de ovelhas mestiças lanadas. Provavelmente, a estacionalidade reprodutiva de animais mestiços lanados, sem raça definida, seja mais influenciada pelas condições de nutrição e manejo, do que efetivamente pelo fotoperíodo. Desta maneira, os protocolos hormonais apresentam-se como boa alternativa a ser utilizada em ovelhas mestiças lanadas e deslanadas durante o período da contraestação reprodutiva.

\section{Agradecimentos}

Ao Conselho Nacional de Desenvolvimento Científico e Tecnológico (CNPq) e ao Programa de Pós-Graduação em Ciência Animal da Universidade Estadual de Londrina pelo financiamento do experimento, e à empresa Tecnopec, pela doação dos hormônios utilizados.

\section{Referências}

ANUÁRIO DO OBSERVATÓRIO NACIONAL. Disponível em: <http://euler.on.br/ephemeris/index. php>. Acesso em: 20 maio 2009.

AYRES, M.; AYRES JUNIOR, M.; AYRES, D. L.; SANTOS, A. S. BioEstat 5.0 - aplicações estatísticas nas áreas das ciências biológicas e médicas. Brasília: Sociedade Civil Mamirauá, CNPq, 2007. 138 p.

CALDEIRA, R. M.; VAZ PORTUGAL, A. Condição corporal: conceitos, métodos de avaliação e interesse da sua utilização como indicador na exploração de ovinos. Revista Portuguesa de Ciências Veterinárias, Lisboa, v. 93, n. 526, p. 95-102, 1998.

CLOETE, S. W. P.; SNYMAN, M. A.; HERSELMAN, M. J. Productive performance of Dorper sheep. Small Ruminant Research, Amsterdam, v. 36, n. 2, p. 119-135, 2000.

COLÉGIO BRASILEIRO DE REPRODUÇÃO ANIMAL - CBRA. Manual para exame e avaliação de sêmen animal. Colégio Brasileiro de Reprodução Animal. 2. ed. Belo Horizonte: CBRA, 1998, 79 p.

COSTA, M. J. R. P.; QUEIROZ, S. A.; RIBEIRO, J. L. C.; RODRIGUES, M. J. P. C.; COSTA, J. L. R.; QUEIROZ, $\mathrm{S}$. Evaluation of some aspects of the performance of Morada Nova sheep in Franca, São Paulo. Revista da Sociedade Brasileira de Zootecnia, Viçosa, v. 19, n. 4, p. 340-346, 1990.

DIAS, F. E. F.; LOPES JUNIOR, E. S.; VILLAROEL, A. B. S.; RONDINA, D.; LIMA-VERDE, J. B.; PAULA, N. R. O.; FREITAS, V. J. F. Sincronização do estro, indução da ovulação e fertilidade de ovelhas deslanadas após tratamento hormonal com gonadotrofina coriônica eqüina. Arquivo Brasileiro de Medicina Veterinária e Zootecnia, Belo Horizonte, v. 53, n. 5, p. 618-623, 2001.

DOGAN, I.; NUR, Z. Different estrous induction methods during the non-breeding season in kivircik ewes. Veterinary Medicine, Czechoslovakia, v. 51, n. 4, p. 133-138, 2006. 
ELIAS, E.; COHEN, D.; DAYENOFF, P. Characteristics and indices of reproduction in Dorper sheep. Journal of the South African Veterinary Association, Pretoria, v. 56, n. 3, p. 127-130, 1985.

FIGUEIREDO, E. A. D.; OLIVEIRA, E. R.; BELLAVER, C. Performance dos ovinos deslanados no Brasil. Sobral: EMBRAPA, CNPC, 1980. $32 \mathrm{p}$.

FIGUEIREDO, E. L.; NUNES, J. F.; CORDEIRO, M. A.; SOUZA, P. T.; DIÓGENES FILHO, R. N.; VIEIRA, V. E.; SILVA FILHO, A. H. S.; MESQUITA, F. L. T.; SALGUEIRO, C. C. M.; FEITOSA, J. V. Inseminação artificial de ovelhas da raça Santa Inês com sêmen diluído em água de coco in natura e em pó. Revista Brasileira de Ciência Veterinária, Niterói, v. 14, n. 2, p. 95-97, 2007.

FONSECA, J. F. Estratégias para o controle do ciclo estral e superovulação em ovinos e caprinos. In: GONGRESSO BRASILEIRO DE REPRODUÇÃO ANIMAL, 16., 2005, Goiânia. Palestras... Goiânia: Colégio Brasileiro de Reprodução Animal, 2005, p. 1-9.

FUKUI, Y.; YAMAMOTO, Y.; GODA, S.; ONO, H. Single or double insemination at fixed-time basis on labing rate of ewes treated with progestogen-impregnated intravaginal sponge during the non-breding season. Japanese Journal of Animal Reproduction, Tokyo, v. 37, p. 231-235, 1991.

GATENBY, R. M. Sheep production in the tropics and sub-tropics. Longman: Condon. 1986. 350 p.

GIRÃO, R. N.; MEDEIROS, L. P.; GIRÃO, E. S. Índices produtivos de ovinos da raça Santa Inês no Estado do Piaui. Teresina: EMBRAPA, UEPAE, 1984. 5 p.

GODFREY, R. W.; COLLINS, J. R.; HENSLEY, E. L.; WHEATON, J. E. Estrus synchronization and artificial inseminated of hair sheep ewes in the tropics. Theriogenology, New York, v. 51, n. 5, p. 985-97, 1999.

GRECCO, B.; MONREAL, A. C. D. Aparecimento do ESTRO após o uso de ECG em dois momentos distintos da sincronização em ovinos. In: CONGRESSO BRASILEIRO DE MEDICINA VETERINÁRIA, 35., 2008, Gramado. Anais... Gramado: CONBRAVET, 2008.

HAFEZ, E. S. E.; JAINUDEEN, M. R.; WAHID, H. Ovinos e caprinos. In: HAFEZ, E. S. E.; HAFEZ, B. (Ed.). Reprodução Animal. 7. ed. São Paulo: Manole, 2004. p. 173-192.

HENDERSON, D. C.; DOWNING, J. M.; BECK, N. F. G. Oestrus synchronization in ewes: a comparison of prostaglandin F2 than salt with a progestagen pessary. Animal Production, Cambridge, v. 39, n. 2, p. 229-233, 1984.

IIDA, K.; KOBAYASHI, N.; KOHNO, H.; MIYAMOTO,
A.; FUKUI, Y. A comparative study of induction of estrus and ovulation by tree different intravaginal devices in ewes during the nonbreeding season. Journal of Reproduction and Development, Tokyo, v. 50, p. 6369, 2004.

KOHNO, H.; OKAMOTO, C.; IIDA, K.; TAKEDA, T.; KANEKO, E.; KAWASHIMA, C.; MIYAMOTO, A; FUKUI, Y. Comparison of estrus induction and subsequent fertility with two different intravaginal devices in ewes during the nonbreeding season. Journal of Reproduction and Development, Tokyo, v. 51, n. 6, p. 805-812, 2005.

KUSAKARI, N.; OHARA, M.; MORI, Y. Seasonal variation in the timing of estrus behavior, LH surge and ovulation following the treatment with progesterone and PMSG in Suffolk ewes. Journal of Reproduction and Development, Tokyo, v. 41, n. 4, p. 212-249, 1995.

MORI, R. M.; RIBEIRO, E. L. A.; MIZUBUTI, I. Y.; ROCHA, M. R.; SILVA, L. D. F. Desempenho reprodutivo de ovelhas submetidas a diferentes formas de suplementação alimentar antes e durante a estação de monta. Revista Brasileira de Zootecnia, Viçosa, v. 35, n. 3, p. 1122-1128, 2006.

MUFTI, A. M.; WANI, G. M.; WANI, N. A. Superovulatory response in Corriedale sheep during different months of the breeding season. Small Ruminant Research, Amsterdam, v. 25, n. 2, p. 181-184, 1997.

NUNES, J. F.; FIGUEIRÓ, P. R. P. Fatores que afetam o comportamento reprodutivo em ovelhas Corriedale e Polwarth. Ciência Rural, Santa Maria, v. 5, n. 4, p. 301307, 1975.

OZYURTLU, N.; KUCUKASLAN, I.; CETIN, Y. Characterization of oestrous induction response, oestrous duration, fecundity and fertility in awassi ewes during the non-breeding season utilizing both cidr and intravaginal sponge treatments. Reproduction in Domestic Animal, New York, v. 45, p. 464-467, 2010.

PRUCOLLI, J. O.; BACCARI JUNIOR, F. L. Estudos sobre estação de monta em ovinos no Estado de São Paulo. Boletim da Indústria Animal, Nova Odessa, v. 24, p. 75-80, 1967.

RIBEIRO, E. L. A.; ROCHA, M. A.; SILVA, L. F. Aspectos reprodutivos em ovelhas Hampshire Down submetidas à monta contínua na região Norte do Paraná. Revista Brasileira de Zootecnia, Viçosa, v. 25, n. 4, p. 637-646, 1996.

RODA, D. S.; SANTOS, L. E.; CUNHA, E. A.; BIANCHINE, D.; FEITOZA, A. S. L.; SANCHEZ, R. D.; LEAL, F. A. Performance of ewes mated at intervals of eight months. Boletim da Indústria Animal, Nova 
Odessa, v. 50, n. 1, p. 49-54, 1993.

RODRIGUES, M. R. C.; ARAÚJO, A. A.; SOUZA, A. L.; ARRUDA, I. J.; LIMA, I. M. T.; LIMA NETO, J. M.; RONDINA, D. Resposta à sincronização do estro e taxa de prenhez de ovelhas santa inês submetidas à suplementação alimentar com bagaço de caju desidratado (Anacardium Occidentale). In: CONGRESSO BRASILEIRO DE MEDICINA VETERINÁRIA, 35., 2008, Gramado. Anais... Gramado: CONBRAVET, 2008.

ROSA, H. J. D.; BRYANT, M. J. Seasonality of reproduction in sheep. Small Ruminant Research, Amsterdam, v. 48, p. 155-171, 2003.

SALAZAR, W. V. Uso do eCG em ovelhas Suffolk no periodo de transição estacional. 2006. Dissertação (Mestrado) - Universidade Federal de Mato Grosso do Sul. Faculdade de Medicina Veterinária e Zootecnia, Campo Grande.

SANTOS, G. M. G.; SILVA, K. C. F.; CASIMIRO, T. R.; COSTA, M. C.; MORI, R. M.; MIZUBUTI, I. Y.; MOREIRA, F. B.; SENEDA, M. M. Reproductive performance of ewes mated in the spring when given nutritional supplements to enhance energy levels. Animal Reproduction, Belo Horizonte, v. 6, n. 2, p. 422-427, 2009.

SASA, A.; TESTON, D. C.; CRIVELLENTI, T. L.; RODRIGUES, P. A.; SILVA, E.C. F.; COELHO, L. A.; SCHALCH, E. Concentrações plasmáticas de progesterona em borregas lanadas e deslanadas durante o período de abril a novembro no Estado de São Paulo. Revista Brasileira de Zootecnia, Viçosa, v. 31, n. 3, p. 1150-1156, 2002.

SASA, A.; TESTON, D. C.; SILVA, E. C. F.; CRIVELLENTI, T. L.; PORTO, M. S. C. S.; RODRIGUES, P. A.; COELHO, L. A. Perfil plasmático de progesterona e incidência mensal de ovulações silenciosas em borregas lanadas e deslanadas criadas no Estado de São Paulo. In: CONGRESSO BRASILEIRO DE ZOOTECNIA - ZOOTEC, 11., 2001, Goiânia. Anais... Goiânia: ZOOTEC, 2001. p. 16.

SILVA, A. E. D. F.; FOOTE, W. C.; RIERA, S. G.; UNANIAN, M. M. Efeito do manejo nutricional sobre a taxa de ovulação e folículos, no decorrer do ano, em ovinos deslanados no Nordeste do Brasil. Pesquisa Agropecuária Brasileira, Brasília, v. 22, n. 6, p. 635-645, 1987.

SILVA, A. E. D. F.; NUNES, J. F. Estacionalidade na atividade sexual e qualidade do sêmen nos ovinos deslanados das raças Santa Inês e Somalis Brasileira. Sobral: EMBRAPA, CNPC, 1987. 14 p.
SILVA, B. D. M. Sincronização de estro com prostaglandina $F 2 \alpha$ versus progesterona associada à gonadotrofina coriônica equina (eCG) em ovelhas deslanadas no Distrito Federal. Brasília, 2008. Dissertação (Mestrado) - Faculdade de Agronomia e Medicina Veterinária, Universidade de Brasília, Brasília.

SILVA, O. L.; FIGUEIRÓ, P. R. P. Efeito da época de cobertura sobre a fertilidade de ovelhas e mortalidade de cordeiros na raça Corriedale. In: REUNIÃO ANUAL DA SOCIEDADE BRASILEIRA DE ZOOTECNIA, 17., 1980, Fortaleza. Anais... Viçosa, MG: Sociedade Brasileira de Zootecnia, 1980. p. 127.

SIMPLÍCIO, A. A.; RIERA, G. S.; NELSON, E. A.; PANT, K. P. Seasonal variation in the seminal and testicular characteristics of Brazilian Somalis rams in the semi-aris climate of tropical northeast Brazil. Journal of Reproduction and Fertilility, Cambridge, v. 66, n. 2, p. 735-738, 1982.

TAKADA, L.; BICUDO, S. D.; RODRIGUES, C. F. C.; LENZ, F. F.; BIANCHINI, D. Avaliação dos momentos do início do estro e da ovulação em ovelhas Suffolk submetidas a protocolo de curta duração para a sincronização do estro na pré-estação reprodutiva. Revista Brasileira de Reprodução Animal, Belo Horizonte, v. 27, n. 3, p. 475-477, 2003.

UNGERFELD, R.; RUBIANES, E. Short term primings with different progestogen intravaginal devices (MAP, FGA and CIDR) for eCG-estrous induction in anestrus ewes. Small Ruminant Research, Amsterdam, v. 46, p. 63-66, 2002.

VILLARROEL, A. B. S. Perdas reprodutivas dos ovinos no Brasil. Revista Brasileira de Reprodução Animal, Belo Horizonte, p. 252-257, 1991.

ZARAZAGA, L. A.; MALPAUX, B.; CHEMINEAU, P. Amplitude of the plasma melatonin nycthemeral rhythms is not associated with the dates of onset and offset of the seasonal ovulatory activity in the Ile-de-France ewe. Reproduction, Nutrition and Development, Paris, v. 43, p. 167-177, 2003. 
